\title{
Mapeamento Cerebral
}

\author{
Délrio Façanha Silva*, Márcia Marques Lima**, Renato Anghinah**e \\ José Geraldo de Camargo Lima***
}

\section{RESUMO}

O mapeamento cerebral é uma técnica digital que gera mapas topográficos coloridos da atividade eletrencefalográfica captada sobre 0 escalpo. 0 eletrencefalograma após passar por um microcomputador, que realiza a análise quantitativa em várias faixas de frequiência, pode ser visualizado em um monitor colorido (EEG digital). Posteriormente, as épocas do EEG digital são selecionadas para a realização dos mapas, impressão final e arquivo em disquetes. Este método tem demonstrado ser útil em várias doenças neurológicas e psiquiátricas e deve ser realizado sempre em conjunto com o EEG digital. Achamos que dentro de alguns anos os aparelhos de EEG digital/mapeamento cerebral substituam as máquinas que realizam o EEG convencional.

\section{UNITERMOS}

Mapeamento cerebral. EEG digital. Eletrencefalografia.

Doutor em Neurologia pela Escola Paulista de Medicina (EPM); Chefe do Setor de Eletrencefalografia (EEG) da Escola Paulista de Medicina (EPM) da Universidade Federal de São Paulo (UNIFESP).

** Médicos estagiários do Setor de EEG da EPM (UNIFESP).

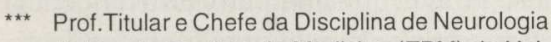
da Escola Paulista de Medicina (EPM) da Universidade Federal de São Paulo (UNIFESP).
Nas últimas décadas o grande avanço tecnológico, principalmente na área da informática, tornou possível a automatização de uma série de atividades que, quando exercidas pelo homem, são sujeitas às suas limitações intrínsecas. Na neurologia, algumas técnicas de imagem foram favorecidas por esta evolução da computação, como, por exemplo, a tomografia computadorizada (TC), a ressonância magnética (RM), a tomografia por emissão de pósitrons (PET), o SPECT ("single photon emission computed tomogra-phy") e a topografia eletrencefalográfica computadorizada. Esta última é mais conhecida como mapeamento, mapeamento cerebral ou "brain mapping" e chamada de cartografia cerebral por algumas escolas francesas.

A análise topográfica do EEG tem suas raízes no toposcópio, descrito por Walter e Shipton (1951) e nos estudos iniciais sobre os topogramas realizados por Rémond e Torres (1964), Rémond (1968) e Ragot \& Rémond (1978). Os primeiros mapeamentos cerebrais foram descritos na Europa em 1971 por Lehmann, seguido por Ueno e Matsuoka (1976), no Japão, e Duffy et al. (1979) nos Estados Unidos da América do Norte (EUA). A partir da década de 1980, com o auxílio da teconologia dos computadores e dos monitores coloridos, o mapeamento se tornou um exame conhecido e muito utilizado. Suas primeiras aplicações clínicas foram descritas nos EUA (Buchsbaum et al., 1982; Duffy et al., 1979; Harner \& Ostergren, 1976), posteriormente no Japão ("Conferência Japonesa de Eletrencefalografia Topográfica", 1983) e, em seguida, na Europa (Persson \& Hjorth, 1983). No Brasil, o interesse pelo método vem aumentando (Calistro Balestrassi et al., 1991). Desde maio de 1994, a Disciplina de Neurologia da Escola Paulista de Medicina da Universidade Federal de São Paulo (UNIFESP), conta com o equipamento de EEG digital (Figura 1) / mapeamento cerebral (Figura 2) (NeuroMap - NeuroTec), que tem sido de grande utilidade na rotina, pesquisa e ensino.

Devido às suas numerosas aplicações, existe o risco do uso e avaliação imprópria (Binnie \& MacGillivray, 1992; Kahn et al., 1988; Nuwer, 1989, 1991) e recomendações têm sido delineadas para evitar esses contratempos (American Electroencephalography Society, 1987; Duffy, 1989; Hermann et al., 1989; Nuwer, 1988b).

Nesta breve revisão, abordaremos apenas alguns aspectos práticos, a utilidade do método em diferentes situações clínicas e não faremos comentários a respeito dos potenciais evocados e métodos estatísticos.

\section{DEFINIÇÃO}

O mapeamento cerebral (MC) é a representação através de gráficos de um mapa elaborado pelo computador. Todas as informações são provenientes do registro eletrencefalográfico, que é obtido pela captação dos potenciais elétricos cerebrais, após a colocação 


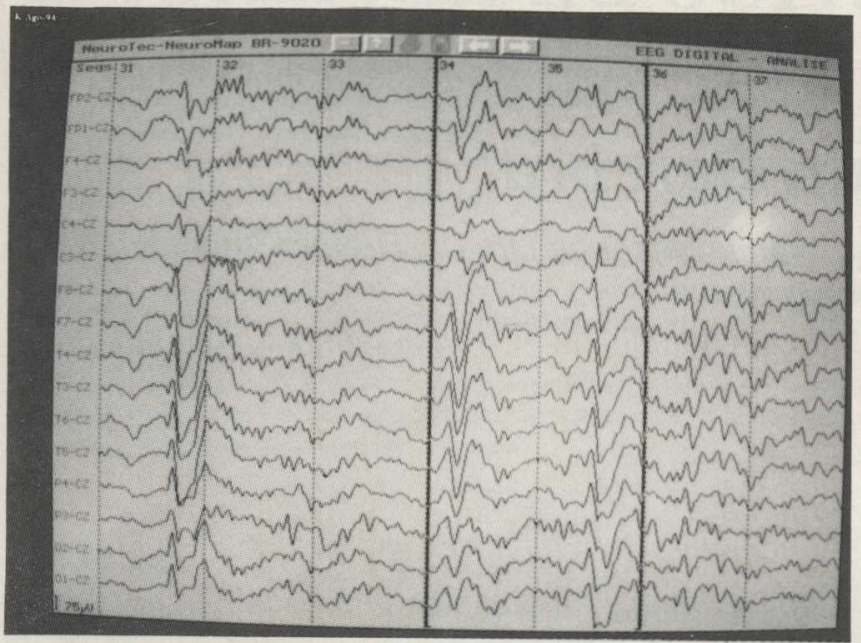

FIGURA 1

Demonstração do EEG na tela do monitor colorido, após ser processado pelo microcomputador.

dos eletrodos no couro cabeludo. Em suma, o MC é uma técnica digital que forma mapas topográficos coloridos da atividade eletrencefalográfica captada sobre o escalpo.

\section{TÉCNICA}

Após a colocação dos eletrodos na superfície craniana, de acordo com o sistema internacional 10-20 (Jasper, 1958), os potenciais elétricos cerebrais são captados e amplificados por um sistema clássico de EEG. Entretanto, antes da passagem desses potenciais para as etapas seguintes do exame, são submetidos a um tratamento matemático pela trasformação rápida de Fourier. Durante a realização do exame, o EEG é exibido na tela do monitor (EEG digital) (Figura1) e, após o seu término, pode ser gravado em disquetes. Encerrado o registro do eletrencefalograma, realiza-se a leitura e seleciona-se os melhores momentos para o MC, ou seja, este só é realizado após a escolha dos fragmentos do EEG digital. Desta forma, é fundamental a correta seleção dos melhores momentos, pois todos os passos seguintes durante a realização do mapeamento, serão baseados no fragmento escolhido. Uma escolha inadequada pode levar a uma interpretação e conclusão errôneas do exame. Sempre que necessário, volta-se ao EEG, para que seja descartada a possibilidade de artefatos, correlacionar achados e para revisar dados. Do mesmo modo que o EEG digital, os mapas podem também ser arquivados em disquetes. Após o término do EEG digital e do MC, seleciona-se as amostras para a impressão final.

Em suma, o mapeamento cerebral é uma extensão do eletrencefalograma, não devendo ser interpretado isoladamente, mas em conjunto com o EEG digital.

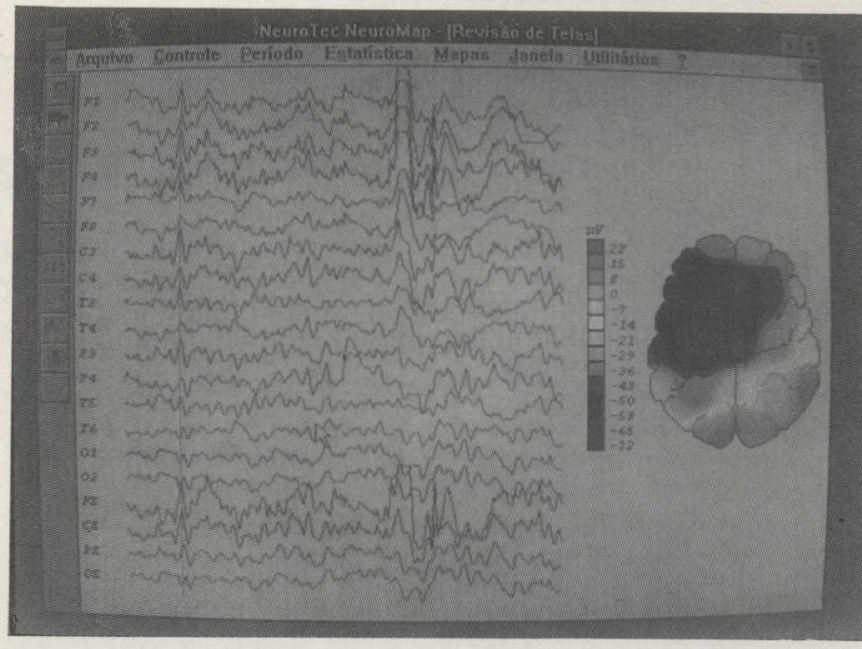

FIGURA 2

Representação gráfica do EEG digital através de mapas elaborados pelo microcomputador.

Após este breve comentário sobre a técnica do EEG digital/MC, passaremos agora a descrever a utilização deste método em diferentes doenças neurológicas e psiquiátricas.

\section{EPILEPSIAS}

A utilidade desta nova técnica no estudo das epilepsias tem apresentado controvérsias. Algumas publicações têm descrito um aumento em todas as faixas de frequiência no local do foco, mesmo nos melhores momentos sem espículas (Gaches \& Guegen, 1989; Harner, 1986; Lombroso \& Duffy, 1982), enquanto outras enfatizam que este aumento é observado principalmente com a atividade beta (Coppola, 1986; Kowell et al., 1987). Entretanto, uma diminuição da atividade rápida também tem sido descrita (Nuwer, 1984, 1988a, c).

Hughes et al. (1991) realizaram o MC em 22 pacientes epilépticos com foco temporal ântero-medial e não-temporal. Selecionaram os melhores momentos sem foco no EEG, foco com raras espículas e foco com freqüentes espículas e "espículas" provocadas por artefatos. Nos fragmentos sem foco, o MC mostrou alterações na região do foco na maioria dos pacientes $(84 \%)$, assim como nas áreas adjacentes $(58 \%)$ e na região homóloga contralateral (foco em espelho) (47\%). Um aumento das atividades delta e beta2 $(16-30 \mathrm{~Hz})$ foi o achado mais comum nos focos mais ativos, enquanto uma diminuição destas atividades foi encontrada nos focos inativos. Quando os melhores momentos com foco foram selecionados, o aumento ocorreu principalmente nas freqüências delta e betal $(12-16 \mathrm{~Hz})$. No MC das "espículas" artefatais, houve um aumento da frequiência delta. Em suma, esse artigo conclui que alguns focos epilépticos, assim como o foco em espelho, podem não ser evidentes 
no EEG convencional, mas sendo identificados no MC, especialmente como delta ou beta2. Entretanto, salientam que qualquer alteração focal no $\mathrm{MC}$ é inespecífica e não pode ser definida como um foco epiléptico, havendo necessidade de mais estudos para serem estabelecidas as diferenças com outras atividades focais. Riggio et al. (1990) obtiveram resultado semelhante ao analisarem os melhores momentos sem foco, no EEG digital, em pacientes com epilepsia do lobo frontal, encontrando alterações no MC em $71 \%$ dos casos estudados.

$\mathrm{O} \mathrm{MC}$ tem sido utilizado no estudo das epilepsias parciais (rolândica), para estabelecer o diagnóstico diferencial entre a epilepsia parcial primária e a secundária (Gregory \& Wong, 1984; Van der Meij et al., 1990, 1992). Van Huffelen e Van der Meij (1990), estudaram através do MC o foco rolândico em crianças com epilepsia parcial primária e com paralisia cerebral. Neste trabalho, foi possível identificar o dipolo tangencial em quase todas (15/20) as crianças com epilepsia rolândica, enquanto o dipolo radial predominou nas crianças com paralisia cerebral (8/13). Entretanto, a identificação do dipolo no EEG digital e MC, caracterizado pela presença do padrão não-estacionário ou dupla ponta-onda, parece ser mais específico das epilepsias parciais primárias, quando comparado com o padrão estacionário (Van der Meij et al., 1992). A identificação destes dipolos é melhor realizada através do EEG digital e do MC que pelo EEG convencional. Apesar da epilepsia rolândica benigna ser uma das mais frequientes da criança, a ocorrência de "status epilepticus" é bem rara (Colamaria et al., 1991; Fejerman \& Blasi, 1987; Roulet et al., 1989; Septien et al., 1992). Recentemente, tivemos a oportunidade de apresentar durante um evento científico (XVI Congresso Brasileiro de Neurologia, Fortaleza - CE, 3 - 8/9/94) o caso de uma criança com este quadro clínico de estado de mal epiléptico e que o estudo realizado com o EEG digital e MC ajudaram na melhor definição do foco.

A maior parte das publicações do estudo do MC nas epilepsias tem se destinado à análise espectral dos ritmos que constituem a atividade elétrica cerebral de base, na tentativa de localizar a região responsável pelo foco epiléptico (Harner, 1986; Harner et al., 1987; Kowell et al, 1987; Lombroso \& Duffy, 1982; Nuwer, 1988a). Entretanto, são raros os trabalhos sobre o estudo topográfico da voltagem do foco temporal (espícula ou onda "sharp") nos pacientes com crise parcial complexa, sendo este método mais utilizado com o MC, na crise generalizada ausência (Rodin \& Ancheta, 1986, 1987). Este tipo de análise não é de fácil realização através do EEG convencional e pode ter importância prática em relação à abordagem cirúrgica dos pacientes com este tipo de crise epiléptica. Ebersole e Wade (1991) estudaram 24 pacientes com crise parcial complexa candidatos à cirurgia. Através do EEG digital e MC caracterizaram dois tipos de espículas nestes pacientes: a) "Tipo 1" - espículas que apresentavam um dipolo caracterizado por uma negatividade na região temporal anterolateral e positividade na região centroparietal contralateral; b) "Tipo 2" - as espículas foram caracterizadas apenas por uma negativida- de na região fronto temporal. Um ou outro tipo predominou em todos os pacientes, exceto em dois que apresentavam simultaneamente tanto o "Tipo 1" como o "Tipo 2". A correlação clínica com EEG intracraniano demonstrou que o "Tipo 1" envolve a região temporal mesial, enquanto o "Tipo 2" o neocórtex frontal ou temporal. A RM mostrou atrofia hipocampal em $82 \%$ dos pacientes com espículas "Tipo 1", enquanto apenas $10 \%$ do "Tipo 2" apresentaram este achado. Esta distinção é clinicamente importante, devido ao sucesso cirúrgico que geralmente é obtido nos pacientes com crise parcial complexa e foco na região temporal mesial (Engel, 1987).

A utilização dos eletrodos esfenoidais é de grande utilidade na localização das descargas epilépticas dos pacientes com epilepsia do lobo temporal (Ives \& Gloor, 1977; King et al., 1986; Sperling \& Engel, 1986), demonstrando geralmente a região de maior atividade epiléptica (Ives et al., 1991). São raros os trabalhos com MC que utilizam este tipo de investigação nos pacientes com crise parcial complexa (Naylor et al., 1988). Em 1993, Ives et al. publicaram uma nova técnica utilizando o MC juntamente com eletrodos esfenoidais, em 40 pacientes com epilepsia do lobo temporal. Concluiram que a visualização topográfica das descargas epilépticas críticas e inter-críticas, através do MC com eletrodos superficiais e esfenoidais, favorece uma melhor análise do exame eletrencefalográfico. No Brasil, esperamos que brevemente os equipamentos de $\mathrm{MC}$ desenvolvam esta tecnologia.

Em um estudo por nós desenvolvido utilizando o EEG convencional, demonstramos que a atividade elétrica cerebral de base alentecida, se correlacionou com as epilepsias graves, ao contrário das descargas epilépticas que se correlacionaram com as epilepsias não-graves (Silva, 1989). Entretanto, na literatura por nós pesquisada, encontramos apenas uma publicação que analisa a atividade de base em pacientes epilépticos através do MC, considerando o tipo de crise epiléptica e os efeitos das drogas anti-epilépticas (Miyauchi et al., 1991). Neste trabalho foram estudados 128 pacientes com epilepsia primária e foi encontrado um grande aumento dos ritmos na frequiência delta, theta, alfal (8 $8.8 \mathrm{~Hz})$ e beta $1(13-19.8 \mathrm{~Hz})$, assim como uma diminuiçã̃o da atividade alfa2 $(9-12.8 \mathrm{~Hz})$, quando comparados com o grupo controle de indivíduos normais; os pacientes com crise parcial simples apresentaram mais ondas lentas do que os com crise generalizada tônico-clônica.

Com relação aos focos da linha média $(\mathrm{Fz}, \mathrm{Cz}$ e Pz) ou de localização parassagital, o MC também pode ser de grande utilidade. Durante um evento científico (XVI Congresso Brasileiro de Neurologia, Fortaleza, 3 - 8/9/94) apresentamos um trabalho realizado em pacientes epilépticos com foco parassagital, e que através da utilização do EEG digital e do MC, foi possível estabelecer em qual hemisfério cerebral se originavam as descargas epilépticas (Figura 2). Neste mesmo encontro, apresentamos também 10 pacientes epilépticos com foco de linha média no EEG convencional e que durante a realização do EEG digital e MC verificou-se que as descargas epilépticas estavam localizadas em um dos hemisférios cerebrais. 


\section{ACIDENTES VASCULARES CEREBRAIS}

O ataque isquêmico transitório (AIT) é definido como um déficit neurológico agudo que regride completamente no prazo de $24 \mathrm{~h}$ (Millikan, 1975) e pode ser indicativo de que um acidente vascular cerebral definitivo possa ocorrer (Loeb, 1980). Entretanto, estudos com TC têm demonstrado que ocorre infarto cerebral em alguns pacientes com AIT (Araki et al., 1983; Candelise et al., 1979), assim como alteração persistente do fluxo sanguíneo cerebral (FSC) (Yonekura et al., 1981) e no EEG (Enge et al., 1980). Estes dados sugerem que a atividade elétrica cerebral de base não se recupera totalmente em $24 \mathrm{~h}$, apesar da regressão dos sintomas e sinais que ocorrem no AIT. Nagata et al. (1984) estudando pacientes com AIT através do MC, encontraram anormalidades unilaterais no MC em $88 \%$ dos pacientes que foram avaliados 2 semanas após o AIT, sendo observado uma queda deste índice para $50-67 \%$ a partir da terceira semana. A TC foi normal em $95 \%$ dos casos. Posteriormente, comparando os resultados obtidos através do $\mathrm{MC}$ e do EEG convencional, encontraram que o primeiro método apresentou uma sensibilidade maior na identificação de assimetrias, enquanto o segundo foi melhor na captação de atividades lentas esporádicas.

Nuwer et al. (1987) em um estudo com MC e TC de pacientes com acidente vascular cerebral isquêmico (AVCI), encontraram anormalidades principalmente às custas da atividade delta em $85 \%$ dos casos (17/20), enquanto este tipo de alteração foi observada em apenas $30 \%$ dos pacientes quando da realização do EEG convencional (6/20). Em uma publicação de Nagata et al. (1982), foi observado uma concordância entre os achados na TC de pacientes com AVCI e a presença de atividade delta no $\mathrm{MC}$ e parece que existe uma melhor correlação entre estes dois exames a partir do quinto dia do AVCI (Etevenon \& Gaches, 1983; Gaches, 1984). Nos acidentes vasculares hemorrágicos (AVCH) as pesquisas são raras, achando-se de maneira genérica que a TC seja a melhor indicação (Gaches \& Fredy, 1986). Em publicações que associam o EEG convencional, TC, RMN e FSC nos AVC (Nuwer, 1988c), o MC tem despertado interesse, pois apresenta boa sensibilidade em relação à localização das lesões, assim como nos estudos que associam o PET (Nagata, 1989; Nuwer, 1988c).

\section{DEMENCIAS}

Na demência pré-senil (DPS) e senil (DS) do tipo Alzheimer, os achados eletrencefalográficos mais comumente descritos têm sido uma atividade elétrica cerebral de base alentecida às custas de ondas theta e delta, com ausência do ritmo alfa (Brenner et al., 1986; Coben et al., 1985). Entretanto, esses achados são de pacientes que se encontram em um estágio moderado ou grave da doença. Naqueles pacientes em fase inicial da demência, o EEG convencional geralmente é dado como normal (Duffy et al., 1984b). Porém, é possível que a atividade elétrica cerebral de base desses pacientes contenha muito mais informações, que não são possíveis de serem identificadas apenas pela análise visual do EEG convencional. Duffy et al. (1984a) estudando pacientes com DPS e DS do tipo Alzheimer, no estágio inicial, observaram que no MC havia um predomínio de ondas theta e diminuição da atividade beta (sincronização) na região temporal posterior direita, temporal média e anterior esquerda nos casos de DPS, enquanto o grupo controle, constituído por indivíduos normais, apresentou aumento da atividade beta e diminuição dos ritmos lentos (dessincronização). No grupo de pacientes com DS, houve um aumento na freqüência theta e delta e diminuição da beta, nas regiões frontais e mésio-frontais anteriores, bilateralmente, enquanto no grupo controle ocorreu uma dessincronização. Apesar da DPS e DS apresentarem achados semelhantes no MC (sincronização), a distribuição topográfica foi diferente. Esses resultados com o MC tentam explicar diferenças clínicas e fisiopatológicas entre estas duas entidades. Outras publicações também têm demonstrado a importância do MC no estudo da DPS e DS do tipo Alzheimer (Ihl et al., 1989b), utilizando o FSC (Ihl et al., 1989a) ou o PET (Breslau et al., 1989).

Nas demências decorrentes da doença de Pick, dos múltiplos infartos, do Parkinson e da infecção luética, estudos com MC também têm sido utilizados na tentativa de estabelecer o diagnóstico diferencial entre estas patologias (Maurer \& Dierks, 1991). Nos casos de hidrocefalia de pressão normal, somos de opinião que a TC e a RMN são mais importantes que o MC. Por outro lado, na doença de Creutzfeldt-Jakob o EEG digital e MC são de maior valor diagnóstico que os exames neuroradiológicos.

\section{TUMORES CEREBRAIS}

Estudos com EEG convencional nos tumores cerebrais têm como objetivo principal, estabelecer a correlação entre o achado da localização das anormalidades no EEG e o local do tumor (Fisher-Williams et al., 1962; Hess, 1961), sendo sugerido que geralmente as alterações do EEG coincidem com a topografia expansiva (Decker \& Knott, 1972; Hess, 1961; Kershman et al., 1949). Entretanto, esta correlação de localização entre o EEG e o tumor cerebral varia entre as publicações (Afif et al., 1965; Murphy et al., 1976). Métodos que utilizam a análise automática quantitativa da atividade elétrica cerebral de base do EEG (Gotman et al., 1975), têm demonstrado maior precisão que o EEG convencional na localização dos tumores cerebrais.

Nagata et al. (1985), estudando 15 pacientes com tumores cerebrais malignos através do MC e TC, demonstraram que a análise pelo MC, utilizando uma técnica baseada na divisão do índice das frequiências (delta e theta/alfa e beta), foi mais sensível que a TC para avaliar o estado clínico do paciente. Além disso, a melhora ou piora do EEG foi mais óbvia no EEG digital/MC que no EEG convencional. Concluem, que esta 
técnica através do MC, deve ser utilizada conjuntamente com os exames neuroradiológicos na monitorização dos pacientes com tumores cerebrais.

Os exames neuroradiológicos, como a TC e principalmente a RMN, já demonstraram sua eficiência nos processos expansivos ao nível do tronco cerebral e na fossa posterior, enquanto o MC mostra apenas as conseqüências destas lesões a nível hemisférico (Gaches \& Fredy, 1986). Entretanto, alguns autores acham que mesmo assim o MC pode ser útil no seguimento destes pacientes (Duffy, 1985), enquanto outros são da opinião que mais estudos sistemáticos são necessários para que se possa tirar conclusões a respeito (Lopes da Silva, 1990).

\section{DOENÇAS PSIQUIÁTRICAS}

Os trabalhos com MC, principalmente a análise quantitativa, não são raros no estudo das doenças psiquiátricas, como demonstrou Nuwer (1988c) em uma revisão sobre este assunto. Schatzberg et al. (1986), utilizando o MC, encontraram alentecimento na região temporal posterior direita e aumento da frequiência beta na região frontal bilateral, em pacientes depressivos quando comparados com um grupo controle de normais. Entretanto, Hooshmand et al. (1987) não são da mesma opinião, pois o MC no seu trabalho foi normal tanto nos indivíduos normais como nos depressivos.

Com relação à esquizofrenia, os dados obtidos com o $\mathrm{MC}$ não são de fácil interpretação e a opinião comum é de que o emprego desta técnica não é útil para o diagnóstico (Fisch \& Pedley, 1989), mas pode servir à pesquisa neurofisiológica nesta área (Nuwer, 1989; Pfurtscheller, 1989). Entretanto, esta opinião talvez seja decorrente do fato de haver dentro do grupo de pacientes esquizofrênicos vários subgrupos. Em apoio a este ponto de vista, John et al. (1989) encontraram consideráveis diferenças entre os MC obtidos de dois grupos de pacientes esquizofrênicos. Esses achados de John et al. (1989) indicam que a análise quantitativa topográfica pode oferecer novas possibilidades para uma melhor caracterização dos subtipos de esquizofrenia, com consequiências para o tratamento e prognóstico.

Em suma, um dos campos mais polêmicos da utilização do MC é a psiquiatria, sendo necessário realizar estudos mais prolongados antes de serem tiradas conclusões prematuras sobre a utilidade deste método.

\section{OUTRAS DOENÇAS NEUROLÓGICAS}

Na síndrome de Gilles de la Tourette, a freqüência de EEG anormais varia de 12 a $95 \%$ (Verma et al., 1986), entretanto, outras publicações que utilizam o EEG digital e MC, não mostram diferenças entre esta síndrome e grupo controle de normais (Neufeld et al., 1989).
Nuwer (1988c), em um estudo com MC, encontrou 55\% de anormalidades em pacientes com enxaqueca, contra apenas $5 \%$ no grupo controle de indivíduos normais.

$\mathrm{Na}$ síndrome de Rett, o MC tem demonstrado intenso alentecimento com presença de ondas theta e delta de alta amplitude, quando comparado com grupo controle (Faienza et al., 1989).

Nos traumatismos crânio-encefálicos, o MC pode ser útil principalmente nos casos de seqüelas, sobretudo quando o EEG convencional, a TC e a RMN forem normais (Hoosmand et al., 1987; Nuwer, 1988c).

Recentemente, durante um evento científico (XVI Congresso Brasileiro de Neurologia, Fortaleza - CE, 3 - 8/9/94) tivemos a oportunidade de apresentar o caso clínico de uma paciente com a sindrome de Kluver-Bucy, onde o EEG convencional foi normal, mas o EEG digital/MC evidenciaram um predomínio da atividade theta nas regiões temporais.

Na SIDA (Síndrome de Imunodeficiência Adquirida), o MC também tem demonstrado sua importância nas encefalopatias precoces, como no caso de um paciente de 69 anos que estava sendo tratado de depressão. O MC conduziu este paciente para uma investigação mais profunda, ao mostrar ondas lentas na região posterior direita (Duffy, 1989).

\section{COMENTÁRIOS FINAIS}

Apesar da polêmica existente sobre o real valor do mapeamento cerebral em diferentes situações clínicas, este é um método que vem tendo boa aceitação pela comunidade científica, pois tem demonstrado sua importância, como muito bem documentado pela literatura.

Nos Estados Unidos da América do Norte, até 1989, já haviam em torno de 400 equipamentos em uso, com uma média anual de 50.000 pacientes realizando este exame. Entretanto, apenas 50\% dos neurologistas que utilizavam esta técnica possuíam o título de especialista em eletrencefalografia (Duffy, 1989). Este quadro demonstra o risco da má utilização deste método e tem recebido críticas da literatura (Binnie \& MacGillivray, 1992; Nuwer, 1991).

Em suma, achamos que o mapeamento cerebral é um método que pode nos fornecer informações clínicas adicionais, desde que seja utilizado conjuntamente com o EEG digital, e é provável que dentro de alguns anos ambos substituam o traçado eletrencefalográfico convencional.

\section{SUMMARY}

The Brain Mapping is a digital technique that generates color topographic of EEG activity from scalp. After entering into a small computer, which calculates the amount of EEG activity in several frequency bands, the EEG can be seen in a color monitor (digital EEG). Then the epochs of the digital EEG are chosen in order to construct the maps, to print and archive in diskketes. These techniques have been applied to a variety of neurologic and psychiatric disorders and should always be done together with the digital EEG. We think that within few years digital "paperless" EEG machines are likely to replace conventional recorders. 


\section{KEY WORDS}

Brain Mapping. Digital EEG. Electroencephalography.

\section{Bibliografia}

1. AFIF, A.K.; MORRISON, R.R.; SAHS, A.L.; EVANS, T.C. - A comparison of Chlormerdin Hg-203 scintiencephaloscanning with neuroradiology and electroencephalography for the localization of intracranial lesions. Neurology (Minneap.), 15:56-63, 1965.

2. AMERICAN ELECTROENCEPHALOGRAPHIC SOCIETY. American Electroencephalographic Society statement on the clinical use of quantitative EEG. J. Clin. Neurophysiol., 4:197, 1987.

3. ARAKI, G.; MIHARA, H.; SHIZUKA, M.; YUNOKI, K.; NAGATA, K.; YAMAGUCHI, K.; MIZUKAMI, M.; TAZAWA, T. - CT and angiographic comparison of patients with transient ischemic attacks, correlation with small infarction of basal ganglia. Stroke, 14:276-280, 1983.

4. BINNIE, C.D.; MacGILLIVRAY, B.B. - Brain mapping: a useful tool or a dangerous toy? J. Neurol. Neurosurg. Psych., 55:527-529, 1992.

5. BRENNER, R.P.; ULRICH, R.F.; SPIKER, D.G.; SCLABASSI, R.J.; REYNOLDS, C.F.; MARIN, R.S.; BOLLER, F. - Computerized EEG spectral analysis in elderly normal, demented and depressed subjects. Electroenceph. clin. Neurophysiol., 64:483-492, 1986.

6. BRESLAU, J.; STARR, A.; SICOTTE, N.; HIGA, J.; BUCHSBAUM, M.S. - Topographic EEG changes with normal aging and SDAT. Electroenceph. clin. Neurophysiol., 72:281-289, 1989.

7. BUCHSBAUM, M.S.; RIGAL, F.; COPPOLA, R.; CAPPELLETI, J.; KING, C.; JOHNSON, J. - A new system for gray-level surface distribution maps of electrical activity. Electroenceph. clin. Neurophysiol., 53:237-242, 1982.

8. CALISTRO BALESTRASSI, L.C.; BALESTRASSI, P.P.; BORGES DA SILVA, L.E.; LAMBERT-TORRES, G.; MUKHEDKAR, D. - A low cost implementation of a beam machine. Proceedings of the annual international conference of the IEEE engineering in medicine and biology society, 13(3):1249-1250, 1991.

9. CANDELISE, L.; PERRONE, P.; SCOTTI, G. - Computer tomographic findings in TIA patients. In: Cerebral Vascular Disease 2, 9th Salzburg Conference. Meyer, J.S.; Lechner, H.; Revich, M. (Eds.). Excerpta Medica, Amsterdam, 1979, p81-86.

10.COBEN, L.A.; DANZIGER, W.; STORANDT, M. - A longitudinal EEG study of mild dementia of Alzheimer type: changes at 1 year and at 2.5 years. Electroenceph. clin. Neurophysiol., 61:101-112, 1985.

11.COLAMARIA, V.; SGRO, V.; CARABALLO, R.; SIMEONE, M.; ZULLINI, E.; FONTANA, E.; ZANETTI, R.; GRIMAU-MERINO, R.; DALLA BERNARDINA, B. - Status epilepticus in benign rolandic epilepsy manifesting as anterior operculum syndrome. Epilepsia, 32:329-334, 1991.

12. COPPOLA, R. - Issues in topographic analysis of EEG activity. In: Topographic mapping of brain electrical activity. Butterworths, Boston, 1986, p339-346.

13.DECKER, D.A. Jr.; KNOTT, J.R. - The EEG in intrinsic supratentorial brain tumors: a comparative evaluation. Electroenceph. cl. Neurophysiol., 33:303-310, 1972.

14.DUFFY, F.H. - Brain electrical activity mapping: clinical applications. Psych. Res., 29:379-384, 1989.

15.DUFFY, F.H. - Clinical value of topographic mapping and quantified neurophysiology. Arch. Neurol., 46:1133-1134, 1986.

16.DUFFY, F.H. - The BEAM method for neurophysiological diagnosis. Ann. N.Y. Acad. Sci., 457:19-34, 1985

17.DUFFY, F.H.; ALBERT, M.S.; McANULTY, G. - Brain electrical activity in patients with presenile and senile dementia of the Alzheimer type. Ann. Neurol., 16:439-448, 1984a.
18.DUFFY, F.H.; ALBERT, M.S.; McANULTY, G.; GARVEY, A.J. Age-related differences in brain electrical activity of healthy subjects. Ann. Neurol., 16:430-438, 1984b.

19.DUFFY, F.H.; BURCHFIEL, J.L.; LOMBROSO, C.T. - Brain electrical activity mapping (BEAM): a method for extending the clinical utility of EEG and evoked potential data. Ann. Neurol., 5:309-321, 1979.

20.EBERSOLE, J.S.; WADE, P.B. - Spike voltage topography identifies two types of frontotemporal epileptic foci. Neurology, 41:1425-1433, 1991.

21.ENGE, S.; LECHNER, H.; LOGAR, C.H.; LADUENER, G. - Clinical value of EEG in transient ischemic attacks. In: EEG and Clinical Neurophysiology. Lechner, H. \& Aranibar, A. (Eds.). Excerpta Medica, Amsterdam, 1980, p173-180.

22.ENGEL, J. Jr. - Outcome with respect to epileptic seizures. In: Surgical treatment of the epilepsies. Engel, J. Jr. (Ed.). Raven Press, New York, 1987, p553-572.

23.ETEVENON, P.; GACHES, J. - Electroencéphalographie quantitative et procédé de cartographie d'images d'EEG quantitative sur ordinateur dans l'étude de la sénescence et des accidents ischémiques cérébraux. In: Médicaments et les maladies cérébro-vasculaires. Cahn, J.; Agnoli, F.; Cohadon, F.; Hoyer, S.; Lechner, H. (Eds.). Eurotext, London-Paris, 1983, 1 vol., p145-155.

24.FAIENZA, C.; CAPONE, C.; SANI, E.; VILLANI, D.; PRATI, G. - EEG mapping in a child with Rett syndrome. Psych. Res., 29:425-426, 1989.

25.FEJERMAN, N.; Di BLASI, M. - Status epilepticus of benign partial epilepsies in children: report of two cases. Epilepsia, 28:351-355, 1987.

26.FISCH, B.J.; PEDLEY, T.A. - The role of quantitative topographic mapping or "neurometrics" in the diagnosis of psychiatric and neurological disorders: the cons. Electroenceph. clin. Neurophysiol., 73:5-9, 1989.

27.FISCHER-WILLIAMS, M.; LAST, S.L.; LYBERI, G.; NORTHFIELD, D.W.C.: clinico-EEG study of 128 gliomas and 50 intracranial metastatic tumours. Brain, 85:1-46, 1962.

28.GACHES, J. - EEG mapping. Examples in regional ischemic brain diseases in clinical practice and methodology. Mal. Medic. Drugs. Dis., 1:245-251, 1984.

29.GACHES, J.; FREDY, D. - Electroencéphalographie quantifiée (cartographie) et tomodensitométrie crânienne. Encyclopédie Médico-Chirurgicale (Paris), Neurologie, $17035 \mathrm{~A}^{50}, 12-1986,8 p$.

30.GACHES, J.; GUEGEN, B. - EEG mapping in epilepsy. In: Topographic brain mapping of EEG and evoked potentials. Springer-Verlag, Berlin, 1989, p249-255.

31.GOTMAN, J.; GLOOR, P.; RAY, W.F. - A quantitative comparison of traditional reading of the EEG and interpretation of computer-extracted features in patients with supratentorial brain lesions. Electroenceph. clin. Neurophysiol., 38:623-639, 1975.

32.GREGORY, D.L.; WONG, P.K. - Topographical analysis of the centro-temporal discharges in benign Rolandic epilepsy of childhood. Epilepsia, 25:705-711, 1984.

33.HACHINSKI, V. - Brain Mapping. Arch. Neurol., 46:1136, 1986.

34.HARNER, R.N. - Clinical application of computed EEG topography. In: Topographic mapping of brain electrical activity. Duffy, F.H. (Ed.). Butterworths, Boston, 1986, p347-356.

35.HARNER, R.N.; JACKEL, R.A.; MAWHINNEY-HEE, M.R.; SUSSMAN, N.M. - Computed EEG topography in epilepsy. Rev. Neurol. (Paris), 143:457-461, 1987.

36.HARNER, R.N.; OSTERGREN, K.A. - Progress in computerized EEG topography (CET). Electroenceph. cl. Neurophysiol., 41:541-542, 1976.

37.HESS, R. - Significance of EEG signs for location of cerebral tumors. Electroenceph. cl. Neurophysiol. Suppl., 19:75-110, 1961

38.HOOSHMAND, H.; DIRECTOR, K.; BECKNER, E.; RADFAR, F. Technical aspects of topographic brain mapping. J. Clin. Neurophysiol., 4:228-229, 1987. 
39.HUGHES, J.R.; TABER, J.E.; FINO, J.J. - The effect of spikes and spike-free epochs on topographic brain maps. Cl. Electroenceph., 22(3):150-160, 1991.

40.IHL, R.; EILLES, C.; FRLICH, L.; MAURER, K.; DIERKS, T.; PERISIC, I. - Electrical brain activity and cerebral blood flow in dementia of the Alzheimer type. Psych. Res., 29:449-452, 1989a.

41.IHL, R.; MAURER, K.; DIERKS, T.; FRLICH, L.; PERISIC, I. - Staging in dementia of the Alzheimer type: topography of electrical brain activity reflects the severity of the disease. Psych. Res., 29:399-401, $1989 \mathrm{~b}$.

42.IVES, J.R.; GLOOR, P. - New sphenoidal electrode assembly to permit long-term monitoring of the patient's ictal and interictal EEG. Electroenceph. clin. Neurophysiol., 42:575-580, 1977.

43.IVES, J.R.; GRUBER, L.J.; MAINWARING, N.R. - An "epileptic fingerprint" to identify and classify temporal lobe ictal and interictal discharges using sphenoidal/surface electrodes in a coronal array. Epilepsia, 32 (Suppl 3):95p.1991.

44.IVES, J.R.; ICHIHASHI, K.; GRUBER, L.J.; MAINWARING, N.R.; BLUME, H.W.; SCHOMER, D.L. - New topographic mapping of temporal lobe seizures. Epilepsia, 34(5):890-896, 1993.

45.JAPANESE CONFERENCE OF TOPOGRAPHIC ELECTROENCEPHALOGRAPHY. 1 vol., 1983, 185p.

46.JASPER, H. - The ten-twenty electrode system of the International Federation. Electroenceph. clin. Neurophysiol., 10:371-375, 1958.

47.JOHN, E.R. - The role of quantitative EEG topographic mapping or "neurometrics" in the diagnosis of psychiatric and neurological disorders: the pros. Electroenceph. cl. Neurophysiol., 73:2-4, 1989.

48.KAHN, E.M.; WEINER, R.D.; BRENNER, R.P.; COPPOLA, R. Topographic maps of brain electrical activity - pitfalls and precautions. Biol. Psychiatr., 23:628-636, 1988.

49.KERSHMAN, J.; CONDE, A.; GIBSON, W.C. Electroencephalography in differential diagnosis of supratentorial tumors. Arch. Neurol. Psych., 62:255-268, 1949.

50.KING, D.W.; SO, E.L.; MARCUS, R. - Techniques and applications of sphenoidal recording. J. Clin. Neurophysiol., 3:51-65, 1986.

51.KOWELL, A.P.; REVELER, M.J; NUWER, M.R. - Topographic mapping of EEG and evoked potentials in epileptic patients. J. Clin. Neurophysiol., 4:233-234, 1987

52.LEHMANN, D. - Multichannel topography of human alpha fields. Electroenceph. clin. Neurophysiol., 31:439-449, 1971.

53. LOEB, C. - Protracted transient ischemic attacks. Europ. Neurol., 19:1-11, 1980.

54.LOMBROSO, C.T.; DUFFY, F.H. - Brain electrical activity mapping in the epilepsies. In: Advances in Epileptology. XII Epilepsy International Symposium. Akimoto, H.; Kazamatsuri, J.; Seino, M.; Ward, A. (Eds.). Raven Press, New York, 1982, p173-179.

55. LOPES DA SILVA, F.H. - A critical review of clinical applications of topographic mapping of brain potentials. J. Clin. Neurophysiol. 7(4):535-551, 1990.

56.MAURER, K.; DIERKS, T. - Atlas of Brain Mapping. Maurer, K. \& Dierks, T. (Eds.). Springer-Verlag. 1991, p54-86.

57.MILLIKAN, C.H. - Ad Hoc Committe on cerebrovascular disease. A classification and outline of cerebrovascular disease, part II. Stroke, 6:565-616, 1975.

58.MIYAUCHI, T.; ENDO, K.; YAMAGUCHI, T.; HAGIMOTO, $\mathrm{H}$. Computerized analysis of EEG background activity in epileptic patients. Epilepsia, 32(6):870-881, 1991.

59.MURPHY, J.T.; GLOOR, P.; YAMAMOTO, Y.L.; FEINDEL, W. - A comparison of electroencephalography and brain scan in supratentorial tumors. N. Engl. J. Med., 276:309-313, 1976.

60.NAGATA, K. - Topographic EEG mapping in cerebrovascular disease. Brain Topogr., 2:119-128, 1989.
61.NAGATA, K.; GROSS, C.E.; KINDT, G.W.; GEIER, J.M.; GEOFFREY, R.A. - Topographic electroencephalographic study with power ratio index mapping in patients with malignant brain tumors. Neurosurgery, 17(4):613-619, 1985.

62.NAKATA, K.; KAZUTA, Y.; ARAKI, G.; MIZUKAMI, M. - Topographic electroencephalographic study of transient ischemic attacks. Electroenceph. clin. Neurophysiol., 58:291-301, 1984.

63.NAKATA, K.; MIZUKAMI, M.; ARAKI, G.; KAWASE, T.; HIRANO, M. - Topographic electroencephalographic study of cerebral infarction using computed mapping of the EEG (CME). J. Cereb. Blood Flow Metab., 2:79-88, 1982.

64.NAYLOR, D.E.; LIEB, J.P.; RISINGER, M. - Computer enhancement of scalp-sphenoidal ictal EEG in patients with complex partial seizures. Electroenceph. clin. Neurophysiol., 70:205-219, 1988.

65.NEUFELD, M.Y.; BERGER, Y.; KORCZYN, A.D. - Gilles de la Tourette Syndrome: EEG and Mapping. Psych. Res., 29:427, 1989.

66.NUWER, M.R. - Asymetry of multi-channel evoked potentials in patients with temporal lobe epilepsy. Epilepsia, 25:654, 1984.

67.NUWER, M.R. - Electroencephalographic brain mapping. The West. J. Med., 155(1):67, 1991.

68.NUWER, M.R. - Frequency analysis and topographic mapping of $E E G$ and evoked potentials in epilepsy. Electroenceph. cl. Neurophysiol., 69:118-126, 1988a.

69.NUWER, M.R. - Quantitative EEG: I. Techniques and problem of frequency analysis and topographic mapping. J. Clin. Neurophysiol., 5:1-43, 1988b.

70.NUWER, M.R. - Quantitative EEG: II. Frequency analysis and topographic mapping in clinical settings. J. Clin. Neurophysiol., 5:45-85, 1988c.

71.NUWER, M.R. - Uses and abuses of brain mapping. Arch. Neurol., 46:1134-1135, 1989.

72.NUWER, M.R.; JORDAN, S.E.; AHN, S.S. - Quantitative EEG is abnormal more often than routine EEG in mild stroke. Neurology, 37:369, 1987.

73.PERSSON, A.; HJORTH, B. - EEG topogram: an aid in describing EEG to the clinician. Electroenceph. clin. Neurophysiol., 56:399-405, 1983.

74.PFURTSCHELLER, G. - EEG mapping: current status and future prospects. Med. Prog. T. Tech., 14:89-97, 1989.

75.RAGOT, R.A.; RÉMOND, A. - EEG field mapping. Electroenceph. clin. Neurophysiol., 45:417-421, 1978.

76.RÉMOND, A. - The importance of topographic data in EEG phenomena, and an electrical model to reproduce them. Electroenceph. clin. Neurophysiol. Suppl., 27:27-49, 1968.

77.RÉMOND, A.; TORRES, F. - A method of electrode placement with a view to topographical research. I. Basic concepts. Electroenceph. cl. Neurophysiol., 17:577-578, 1964.

78.RIGGIO, S.; HARNER, R.; HARNER, C. - Topographic EEG analysis of spike and non-spike activity in frontal lobe epilepsy. Electroenceph. clin. Neurophysiol., 75:81p, 1990.

79.RODIN, E.A.; ANCHETA, O.M. - Cerebral electrical fields in patients with epilepsy (abstract). Epilepsia, 27:642, 1986.

80.RODIN, E.A.; ANCHETA, O.M. - Cerebral electrical fields during petit mal absences. Electroenceph. clin. Neurophysiol., 66:457-466, 1987.

81.ROULET, E.; DEONNA, T.; DESPLANT, P.A. - Prolonged intermittent drooling and oromotor dyspraxia in benign childhood epilepsy with centro-temporal spikes. Epilepsia, 30:564-568, 1989.

82.SCHATZBERG, A.F.; ELLIOT, G.R.; LERBINGER, J.E.; MARCEL, B.; DUFFY, F.H. - Topographic mapping in depressed patients. In: Topographic mapping of brain electrical activity. Duffy, F. H. (Ed.). Butterworths, Boston, 1986, p389-391.

83.SEPTIEN, L.; GRAS, P.; GIROUD, M.; DUMAS, R. - Syndrome bi-operculaire antérieur aigu d'origine critique dans l'épilepsie a paroxysmes rolandiques. Rev. Neurol. (Paris), 11:712-715, 1992. 
84.SILVA, D.F. - Atividade elétrica cerebral de base e gravidade das epilepsias. São Paulo. 1989. (Tese - Doutorado - Escola Paulista de Medicina). 103p.

85.SPERLING, M.R.; ENGEL, J.Jr. - Sphenoidal electrodes. J. Clin. Neurophysiol., 3:63-73, 1986.

86.UENO, S.; MATSUOKA, S. - Topographic computer display of abnormal EEG activities in patients with brain lesions. Digest of the 11th. International Conference on Medical and Biological Engineering. Ottawa, 1976, p218-219.

87.VAN DER MEIJ, W.; VAN HUFFELEN, A.C.; WIENEKE, G.H. - EEG mapping and dipole modelling of rolandic spikes. Clin. Neurophysiol., 20:2s, 1990.

88.VAN DER MEIJ, W.; VAN HUFFELEN, A.C.; WIENEKE, G.H. WILLEMSE, J. - Sequential EEG mapping may differentiate "epileptic" from "non-epileptic" rolandic spikes. Electroenceph. cl. Neurophysiol., 82:408-414, 1992.

89.VAN HUFFELEN, A.C.; VAN DER MEIJ, W. - Topographic EEG analysis in patients with benign focal epilepsy of childhood (BFEC). Acta neurol. belg., 90:183-189, 1990.
90.VERMA, N.P.; SYRIGOU-PAPAVASILIOU, A.; LeWITT, P.A. Encephalographic findings in unmedicated neurologically and intellectually intact Tourette syndrome patients. Electroenceph. clin. Neurophysiol., 64:12, 1986.

91.WALTER, G.; SHIPTON, H.W. - A new toposcopic system. Electroenceph. clin. Neurophysiol., 3:281-292, 1951.

92.YONEKURA, M.; AUSTIN, G.; POLL, N.; HAYWARD, W. - Evaluation of regional cerebral blood flow in patients with transient ischemic attacks and minor stroke. Surg. Neurol., 15:57-65, 1981.

\section{Correspondência:}

Dr. Délrio Façanha da Silva - Escola Paulista de Medicina Universidade Federal de São Paulo (UNIFESP) Disciplina de Neurologia - Setor de Eletrencefalografia Rua Botucatu, 740 - CEP 04023 - 900 - São Paulo - SP 